\title{
Pleural Space Complications from Tuberculous Empyema: A Case Report and Short Literature Review
}

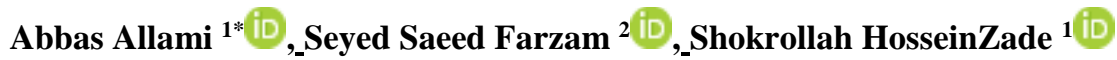 \\ 1. Dept. of Infectious Diseases, Clinical Research Development Unit, BouAli Sina Hospital, Qazvin University of Medical of \\ Sciences, Qazvin, Iran \\ 2. Dept. of Cardiology, BouAli Sina Hospital, Qazvin University of Medical of Sciences, Qazvin, Iran
}

\begin{tabular}{|c|c|}
\hline Article Info & ABSTRACT \\
\hline d 10 10.30699/jambs.28.129.218 & \multirow{4}{*}{$\begin{array}{l}\text { Tuberculous empyema (TE) is an uncommon form of pleural tuberculous (TB). } \\
\text { Although the incidence of TE has significantly decreased, it still threatens public } \\
\text { health. Patients with TE experience a protracted illness and significant morbidity and } \\
\text { mortality risk. Male sex is a significant risk factor for TE. Herein, we report an adult } \\
\text { case of TE admitted to a tertiary care hospital of Iran with clinical characteristics, } \\
\text { treatment modalities, and outcomes of this disease. The duration of illness and } \\
\text { duration of chest tube drainage equaled } 7 \text { months and } 48 \text { days, respectively. Our } \\
\text { patient required surgery despite the administration of antituberculosis drugs and chest } \\
\text { tube drainage. Due to the presence of parenchymal lesions, bronchopleural fistula, } \\
\text { and failure of lung re-expansion after chest tube insertion, the patient required } \\
\text { decortication with the closure of the bronchopleural fistula and additional } \\
\text { thoracoplasty. In the case presentation, we emphasize difficulties encountered in the } \\
\text { treatment of such patients. } \\
\text { Keywords: Bronchopleural fistula, Empyema, Tuberculous empyema }\end{array}$} \\
\hline $\begin{array}{c}\text { Received: 2020/05/11; } \\
\text { Accepted: 2020/07/15; } \\
\text { Published Online: } 31 \text { July 2020; }\end{array}$ & \\
\hline \multirow[t]{3}{*}{$\begin{array}{l}\text { Use your device to scan and read the } \\
\text { article online }\end{array}$} & \\
\hline & \\
\hline & $\begin{array}{l}\text { Corresponding Information: } \\
\text { Abbas Allami, Dept. of Infectious Diseases, Clinical Research Development Unit, BouAli Sina Hospital, } \\
\text { Qazvin University of Medical of Sciences, Qazvin, Iran E-mail: allami9@ yahoo.com }\end{array}$ \\
\hline & $\begin{array}{l}\text { cess article distributed under the terms of the Creative Commons Attribution-noncommercial } 4.0 \text { International License which permits } \\
\text { noncommercial usages with proper citation. }\end{array}$ \\
\hline
\end{tabular}

\section{Introduction}

Tuberculous pleural effusion (TB pleurisy) is the $2^{\text {nd }}$ most common form of extrapulmonary tuberculosis (EPTB) and the most prevalent cause of pleural effusion in endemic tuberculosis areas (1). According to the World Health Organization (WHO), 10 million incident cases of tuberculosis (TB) were estimated worldwide in 2017, 14\% of which were EPTB. In 2015, the incidence rate of tuberculosis was 16 cases per 100,000 people in Iran (2). Tuberculous empyema (TE) was a rare complication of pulmonary tuberculosis in the postchemotherapy era, but it still threatens public health $(3,4)$. Clinical outcomes of TE are generally believed to be worse compared to those of nontuberculous empyema because of protracted illness, presence of concomitant fibrocavitary lung lesions, high bacillary load, development of bronchopleural fistulae (BPF), and requirement of complicated thoracic surgeries in the face of compromised lung function (5). A high risk of poor clinical outcome has been observed in male TE patients, while the concomitant pulmonary TB is protective (4).

The present case study reports the clinical characteristics, outcome, and difficulties in the treatment of a patient with TE. An adult case of nonsurgical thoracic empyema was admitted to the infectious diseases ward of a tertiary care teaching hospital in northern Iran over a period of seven months (Sep 2018-Apr 2019).

\section{Case Report}

The 25-year-old man was admitted with four days of fever, one month of progressive shortness of breath, and four months of weakness, lethargy, anorexia, and a weight loss of $25 \mathrm{~kg}$. He also had coughs, hemoptysis, nausea and vomiting, palpitations, and pleural chest pain. The patient had a two-year history of using heroin and was taking methadone as part of an ongoing opioid dependency treatment and a history of contact with a TB patient in prison. He was married, and his relatives had no recent infectious diseases. He belonged to a low socioeconomic status and lived in a periurban area. The patient had a complete vaccination history of preventable diseases based on immunization programs in the Islamic Republic of Iran. Our patient did not report any defined comorbidities such as diabetes mellitus, human 
immunodeficiency viruses (HIV) infection, hepatitis B and $\mathrm{C}$ virus infections, seizure disorder, and malignancy. Physical examination revealed tachycardia (120/min), fever $\left(39^{\circ} \mathrm{C}\right)$, a reduced right pulmonary sound, and local tenderness. The saturated oxygen level $\left(\mathrm{SO}_{2}\right), \mathrm{pH}, \mathrm{PCO}_{2}$, and bicarbonate were $94 \%, 7.53,28.8 \mathrm{mmHg}$, and 24.4 $\mathrm{mEq} / \mathrm{L}$, respectively. He underwent a lung computed tomography (CT) scan that showed pleural effusion with multiple bullae. In echocardiography, ejection fraction was $55 \%$ and a mild tricuspid valve regurgitation was reported. Normal liver function tests (LFT), normal amylase and lipase, and negative brucellosis and HIV serology were reported. The aspirated pleural fluid was purulent (empyema) (Table 1).

Table 1. Blood gas trends, biochemistry, and complete blood cell count during hospitalization

\begin{tabular}{|c|c|c|c|c|c|c|c|c|c|}
\hline Hospitalization & First & \multicolumn{5}{|c|}{ Second } & \multicolumn{3}{|c|}{ Third } \\
\hline \multicolumn{10}{|c|}{ biochemistry } \\
\hline Creatinine (mg /dl) & 0.9 & 0.8 & - & - & - & - & 0.6 & - & 0.8 \\
\hline $\mathrm{Na}(\mathrm{mEq} / \mathrm{L})$ & 130 & - & - & 136 & - & - & 136 & 136 & 136 \\
\hline $\mathrm{K}(\mathrm{mEq} / \mathrm{L})$ & 4 & - & - & 4 & - & - & 4.4 & 4.4 & 3.8 \\
\hline Blood sugar (mg/dl) & 93 & - & - & - & - & - & 90 & - & 118 \\
\hline \multicolumn{10}{|c|}{ Complete Blood cell count (CBC) } \\
\hline White blood cells (cell $/ \mathrm{mm}^{3}$ ) & 10300 & 7600 & 11300 & 8000 & 9400 & 1500 & 8700 & 7100 & 8500 \\
\hline Neutrophils (\%) & 89 & 86 & 75 & 71 & 87 & 59 & 62 & - & 92 \\
\hline Lymphocytes (\%) & 6 & 9 & 13 & 26 & 6 & 32 & 31 & - & 7 \\
\hline Hemoglobin (mg/dl) & 9.9 & 9.9 & 7.9 & 10.6 & 11 & 9.5 & 11.3 & 14.4 & 13.4 \\
\hline \multicolumn{10}{|c|}{ Inflammatory markers } \\
\hline $\operatorname{ESR}(\mathrm{mm} / \mathrm{h})$ & 92 & 70 & 67 & 80 & 75 & 55 & 88 & 40 & 61 \\
\hline $\mathrm{CRP}(\mathrm{m} \mathrm{g} / \mathrm{l})$ & 90 & 64 & 42 & 49 & 55 & 39 & 59 & 21 & 16 \\
\hline \multicolumn{10}{|c|}{ Liver tests } \\
\hline $\operatorname{AST}(\mathrm{U} / \mathrm{L})$ & 23 & 24 & 39 & 77 & - & - & 15 & 28 & - \\
\hline ALT (U/L) & 7 & 19 & 33 & 117 & - & - & 10 & 14 & - \\
\hline ALKp (U/L) & 313 & - & 267 & 245 & - & - & 243 & 142 & - \\
\hline Bilirubin Direct (mg/dl) & 0.6 & - & 0.4 & - & - & - & 0.3 & 0.2 & - \\
\hline Bilirubin Total (mg/dl) & 1.1 & - & 1.1 & - & - & - & 1 & 0.6 & - \\
\hline
\end{tabular}

Ceftriaxone and clindamycin were started. The acidfast bacilli (AFB) sputum smear result was positive, hence antituberculosis (anti-TB) treatment and vitamin B6 were started, and the patient was transferred to an isolated room. According to the thoracic surgery consultation, no chest tube was required due to the risk of complications. On Day 4, the patient was still febrile, but he was discharged on the same day with anti-TB treatment.

The following day the patient visited the hospital because of severe shortness of breath and pain in the right hemithorax. The patient had noted a dramatic increase in dyspnea over the previous day. His $\mathrm{SO}_{2}$ reached $58 \%$. With a diagnosis of nosocomial infection, intravenous vancomycin and meropenem were started after obtaining the appropriate laboratory workup and blood cultures. Also, the patient was admitted to an isolated room with airborne precaution. A CT scan of the chest without contrast confirmed right lung atelectasis and large right pleural effusion with air-fluid levels (hydropneumothorax, empyema, or abscess) and significant mediastinal displacement. A nodule with an irregular diameter of $41 \mathrm{~mm}$ and hiatal hernias were observed in the left lung (Figure 1).

Two days later, a cardiovascular surgeon (in consultation with a thoracic surgeon) inserted a chest tube that would then remove $500 \mathrm{~mL}$ of the purulent fluid (Figure 2).

In the smear, the exudative fluid was reported to predominate in polymorphonuclear cells (PMN) and in cultures, Pseudomonas was identified (susceptible to ceftazidime, cefepime, ciprofloxacin, amikacin, and gentamicin) (Table 2). 

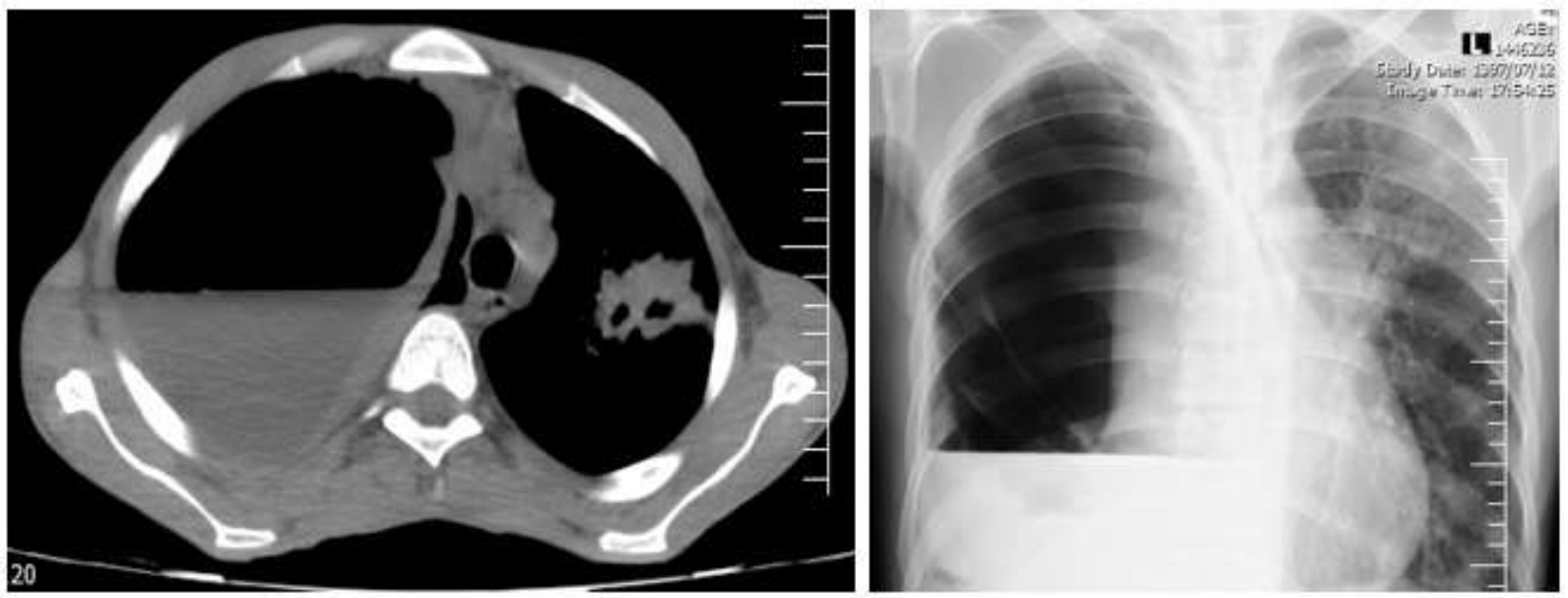

Figure 1. Chest X-ray and CT scan of the patient showing right-sided pyopneumothorax with bilateral parenchymal lesions (the beginning of the second hospitalization)
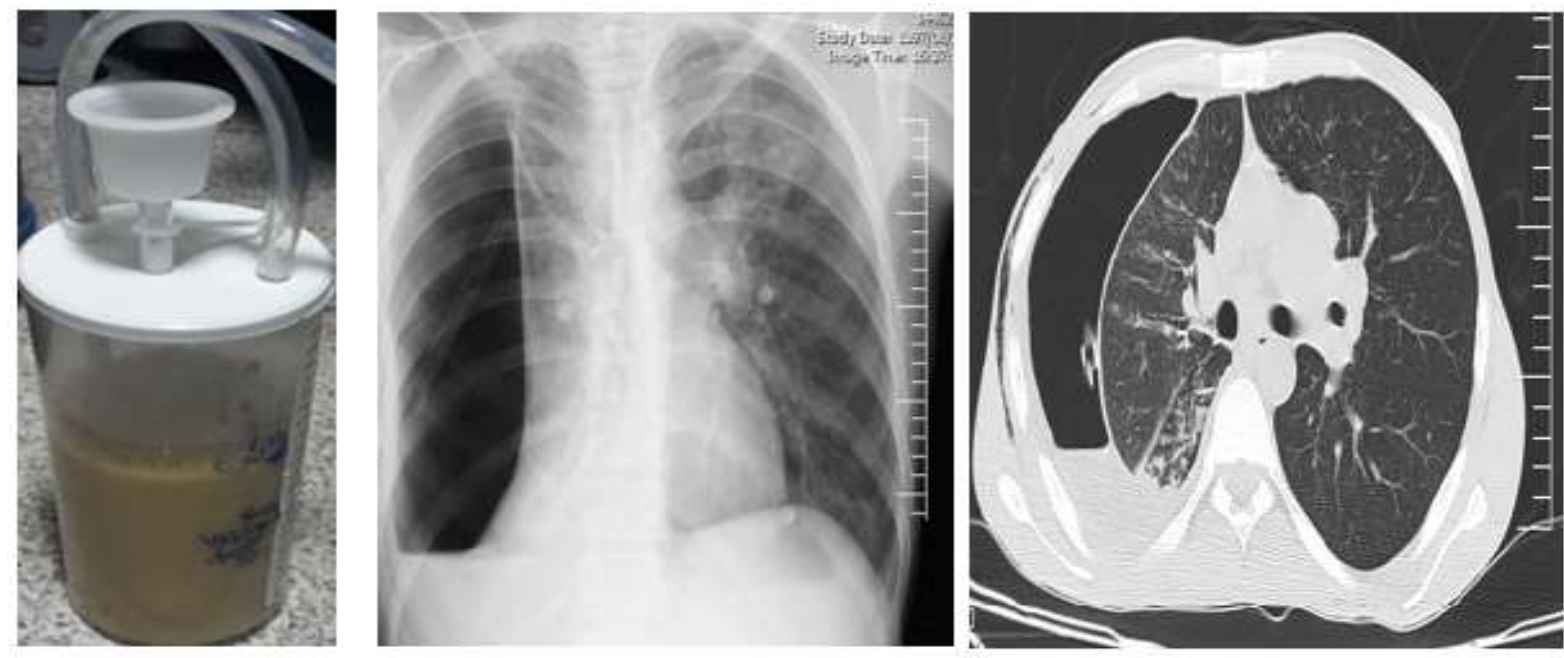

Figure 2. Chest X-ray and CT scan of the patient after the insertion of the chest tube and the drained fluid

Table 2. Trends of changes in the patient's pleural fluid during admission

\begin{tabular}{|c|c|c|c|c|c|}
\hline Hospitalization & First & & Second & & \\
\hline Time (days after admission) & first day & 10 & 20 & 25 & 31 \\
\hline The appearance of the liquid & Purulent & turbid -milky & turbid & - & - \\
\hline $\mathrm{WBC}\left(\mathrm{cell} / \mathrm{mm}^{3}\right)$ & 3100 & 25700 & 14000 & 2400 & 3200 \\
\hline Poly / Lymph & $90 / 10$ & $90 / 10$ & $90 / 10$ & $90 / 10$ & $80 / 20$ \\
\hline $\mathrm{RBC}\left(\mathrm{cell} / \mathrm{mm}^{3}\right)$ & 1250 & 1000 & 1540 & 640 & 800 \\
\hline $\begin{array}{l}\text { Glucose of Pleural fluid } \\
(\mathrm{mg} / \mathrm{dl})\end{array}$ & 92 & 12 & - & - & 12 \\
\hline $\mathrm{Alb}(\mathrm{mg} / \mathrm{dl})$ & 2 & - & - & 0.6 & 2.4 \\
\hline Total protein $(\mathrm{mg} / \mathrm{dl})$ & 4 & 4 & 1.1 & 1 & 3.3 \\
\hline Pleural / Blood Protein & 0.75 & 0.74 & - & - & - \\
\hline LDH (U/L) & 78 & 20 & 1620 & 535 & - \\
\hline Pleural /Blood LDH & 0.18 & 0.05 & - & - & - \\
\hline $\begin{array}{c}\text { Smear and } \\
\text { Pleural cultivation }\end{array}$ & - & $\begin{array}{l}\text { No bacteria in smear } \\
\text { Pseudomonas } \\
\text { in culture }\end{array}$ & $\begin{array}{l}\text { Gram positive cocci } \\
\text { smear } \\
\text { Negative cultivation }\end{array}$ & $\begin{array}{c}\text { No bacteria in } \\
\text { smear } \\
\text { Negative cytology }\end{array}$ & - \\
\hline $\mathrm{ADA}(\mathrm{U} / \mathrm{L})$ & 139 & 460 & - & - & - \\
\hline
\end{tabular}

*MDR: multi drug resistant, ADA: adenosine deaminase 
In control lung CT scans after chest tube insertion, right-sided hydropneumothorax with the fluid-air surface, air bronchogram and a tree-in-bud appearance in the upper segment of the right lower lobe, reduced lung volume and subcutaneous emphysema, and on the left side, opacity and a tree-in-bud view were observed in the upper lobe of the left lung. Air lucency was seen in the upper anterior mediastinum, mostly suggesting tuberculosis (Figure 2).

He was treated with fluconazole several times during the second hospitalization because of oral candidiasis. Due to severe pain in the right upper abdominal area, gallbladder and bile duct ultrasound and LFT were performed. Normal ultrasound and LFT were reported. On several occasions, the patient developed fever, requiring surgical consultation to examine the status of the chest tube and blood culture. Thyroid tests were reported to be normal. Ciprofloxacin and ampi-sulbactam were added to the treatment. In the analysis of the pleural fluid, WBC count and LDH levels increased and pleural fluid protein decreased. The sputum smear was reported negative for AFB several times during the treatment, and thus the patient was transferred to the ward. In the chest X-ray (CXR), mild hydropneumothorax and opacity were detected in the upper and lower lobe of the left lung. However, the patient was still febrile. Two weeks later, the blood culture was negative. In the pleural fluid, the white blood cell (WBC) count and neutrophil percentage decreased.

Three times during the hospitalization period, the patient's chest tube was removed due to fever, leakage of fluid, and twice as a consequence of decreased effusion of the pleural fluid, but was re-implanted due to fluid accumulation and increased distress. Because of abdominal pain and normal ultrasound reports, abdominal and pelvic CT of the abdomen and pelvis was required. Two hypodense regions with an irregular border of $27 \mathrm{~mm}$ in diameter were seen in the spleen, suggesting ischemia or hematoma. Hydropenomotorax was seen on the right lung with atelectasis .. During treatment, the patient developed severe leukopenia (WBC count: 1500 cell $/ \mathrm{mm}^{3}$ ). Wound culture (chest tube insertion site) grew Pseudomonas aeruginosa susceptible to amikacin, ceftazidime, cefepime, ciprofloxacin, and imipenem. Intravenous meropenem was administered. As a result, the patient's leukopenia improved (WBC count reached 3600 cell $/ \mathrm{mm}^{3}$ ), but he was still febrile. Because of the development of nosocomial infection, the patient was treated several times during the hospitalization period. He was transferred to another hospital for thoracic surgery and underwent right partial pneumectomy with ipsilateral rib resection (Figure 3).
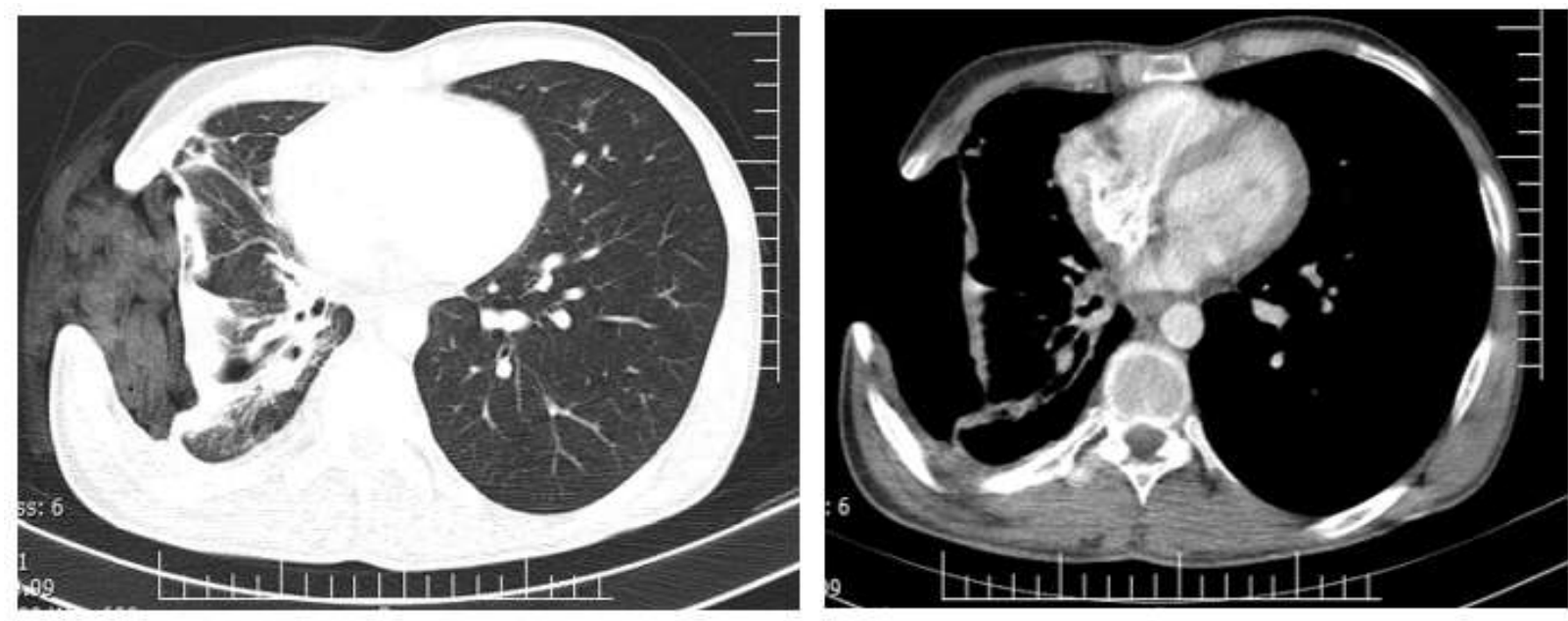

Figure 3. Chest CT scan after thoracoplasty (rib resection and patient decoration)

After three weeks, he was transferred to our hospital and was admitted for the fourth time in one year. At this time, although the chest-tube discharge increased, the sputum smear was negative for AFB. The wound was open with erythema around the chest-tube insertion site but without secretions. In lung CT scans, evidence of changes after the lobectomy surgery, a multibolus image in the right extrapulmonary space, consolidation in the right lower lobe, linear (plate) atelectasis in the right middle lobe, a $32 * 35$-mm-thick, amorphous wall image in the anterior segment, and the upper lobe of the left lung were observed. Cultures of the wound reported Acinetobacter sensitive to amikacin, cefepime, ceftazidime, ciprofloxacin, gentamicin, and imipenem. After the onset of antibiotic treatment and the reduction of the pleural discharge under $50 \mathrm{~mL} / 24$ hours, the patient was discharged with anti-TB medication as well as clindamycin and levofloxacin.

About two months later, the patient returned to our hospital with shortness of breath, headache, fever, coughs, and hot flashes. He had sputum excretion but was unable to get it out. The examination showed a decrease in right and left pulmonary volume and a slight discharge from the site of surgery. His vital signs 
were stable, he was not febrile, and all the tests were normal; hence, he was discharged.

One month later, he returned to the hospital with fever and sweating, shortness of breath, nausea, anorexia, dysuria, coughs and sputum discharge, abdominal pain in the RUQ area, and blurred vision. The examination showed a crackle on the right lung and abdominal tenderness. A $5 * 5-\mathrm{cm}$ sore wound was spotted on the anterior lateral right chest wall with rib resection on it without swelling, tenderness, or discharge. Liver tests were normal, hence anti-TB therapy was continued. Due to vitamin D deficiency, the patient was prescribed vitamin D supplements. He complained of insomnia and depression, as well as aggression and suicidal ideation. According to psychology counseling, antipsychotic medication was initiated and he was transferred to the psychology service. Eventually, the patient was discharged with a good general condition and advised to continue the anti-TB treatment.

\section{Discussion}

The etiology of empyema is determined based on history, physical examination, radiology, and empyema fluid analysis. TE fluid is grossly purulent (1). TE should be considered in patients with risk factors for tuberculous infection. TE is defined as empyema thoracis with one of the following: pleural fluid positive on Ziehl-Nielsen stain, sputum smearpositive for AFB, and having lesions compatible with active parenchymal TB on the CXR/CT scan (6). TE is a rare disease in which TB infection leads to pus in the pleural space where the predominant cell is the PMN. Delayed diagnosis and treatment of TE are associated with poor clinical outcome. TE must be distinguished from tuberculous pleurisy in which a lymphocytic effusion occurs (7). In the case presented here, frank pus was aspirated at thoracentesis, sputum smearpositive for AFB, and having lesions compatible with active parenchymal TB (a tree-in-bud appearance and nodular consolidation with a cavity in the apex), and poly/lymph was 90/10. The primary pleural fluid adenosine deaminase (ADA) level of our patient was 139 units/L and, after 10 days, increased to 460 units/L. A recent meta-analysis has reported that ADA has a favorable diagnostic value in tuberculous pleural effusions (8). Studies have suggested that an ADA level $>60$ units/L is highly sensitive and specific (100\% and up to $97 \%$, respectively) for TB pleurisy (9), and a very high ADA activity is considered in empyema (10). The pleural ADA level might be valuable for establishing a diagnosis of TB pleurisy, even when smear and culture are negative (11).

Our patient was a 25-year-old man. Male preponderance is seen in TE (3). TE usually occurs in younger and middle-aged patients. (12) The majority of TE cases belong to a relatively younger age group (1530 years), whereas patients with nontuberculous empyema are aged $>45$ years. Similar to our case, the majority of TE have an illness with a >1-month duration. The patient was a 25-year-old man. The majority of the TE patients in the study by Hama presented at a young age (15-30 years) and mean age was 33 years. This finding has been supported by other studies, e.g. Acharya et al. (median age: 20-40 years) (13). Crowded institutions and closed communities such as prisons are classical contexts that promote the occurrence of TB. It is expected that the prevalence of TB should be higher among incarcerated patients, as in those with a prison history (14).

Evidence of active pulmonary tuberculosis in the right lobe, thickened parietal pleura, and pyopneumothorax on the lung CT scan was reported. An associated parenchymal lesion in the same and opposite lung was observed. TE is often associated with a thickened, scarred, and calcified pleura (15).

Our patient demonstrated the evidence of coexisting bacterial infection (The Gram-stains and culture of pleural fluid were positive). In microbiological studies, Gram-negative bacilli were isolated. He received a prolonged antibiotic course during admission. Our patient had a positive AFB sputum. From the point of view of pathogenesis, such high mycobacterial smear positivity in the sputum is due to a high bacillary load, which is reflected by the presence of concomitant parenchymal lesions and a bronchopleural fistula, similar to our case (5). BPF was diagnosed with CXR prior to thoracentesis revealing the air-fluid level in the upright position, and the air leak via the tube thoracostomy persisted for $>1$ day after tube thoracostomy. Rupture of a tubercular cavity into the pleural space and persistence of the communication constitute the mechanism of BPF in TE. The likely mechanism of bacterial superinfection in our patient and most TE patients is persistent BPF. Bacterial coinfection with TE has been reported in other studies (6). Our patient was initially put on parenteral antibiotics until determining the results of susceptibility testing and Gram stain/culture. For the initial three weeks, parenteral antibiotics were administered, followed by oral antibiotics.

In patients with an empyema (similar to our case), an empiric, broad-spectrum antibiotic that provides coverage for anaerobic organisms should be initiated promptly, because empyemas often harbor multiple species of bacteria, particularly anaerobes. Management of tuberculous pleural effusion consists of anti-TB therapy. Pleural drainage is usually reserved for patients with severe symptoms (dyspnea); otherwise, routine complete drainage of pleural fluid at the time of diagnosis does not appear to affect longterm outcomes (16). In fact, the approach to anti-TB therapy for the treatment of tuberculous pleural effusion is the approach to the treatment of active pulmonary TB. Therefore, in the primary center, no chest tube was inserted, and the patient only received category I anti-TB treatment. 
In our hospital, the surgeon decided to insert a chest tube due to a dramatic increase in dyspnea, decreased $\mathrm{SO}_{2}$ to $58 \%$, right lung atelectasis, and large right pleural effusion with air-fluid levels and significant mediastinal displacement. Generally, chest tube drainage is preferred over more invasive options for free-flowing fluid and uniloculated effusions. In our case, the drainage modality was intercostal tube drainage (ICTD), but there was a failure of lung expansion even after ICTD. The duration of ICTD was 48 days. Chest tubes are typically left in place until the fluid becomes serous, the drainage rate falls $<50$ $\mathrm{mL} /$ day, the empyema cavity is closed, and BPF is sealed (17).

Despite the progress in TB management, not all TE patients achieve full lung re-expansion and occasionally a pleural space remains after treatment. In our patient, lung compliance and volume were irreversibly reduced (the development of restrictive fibrothorax). In some TE patients, similar to our patient, there may be recurrent infection in the pleural space that needs surgical intervention. In such situations, antimicrobial therapy is often insufficient because of poor drug penetration to the pleural collection. Drainage is also often unsuccessful because the pus is thick. In such cases, the only effective management is surgical drainage and decortication (18). Our patient failed to respond to antibiotic therapy and ICTD and his fever persisted. Surgical drainage was carried out due to loculations and inadequate drainage, the presence of BPF and nonexpansion of the lung. In fact, TE causes the failure of lung re-expansion after ICTD. In our case, there were other problems such as the occurrence of infection due to a leakage of pleural fluid caused by the detachment of the chest tube, and induction of emphysema of the lung due to air infiltration. Decortication with the closure of BPF and thoracoplasty were the three surgical procedures performed. Continued signs of infection and inadequate pleural drainage should prompt thoracoscopy or thoracotomy to optimize chest tube placement and fully drain the pleural space (19). In patients with a larger empyema cavity size, more adhesions, and greater visceral pleural thickness, the surgical procedure of choice is decortication. In other situations, thoracoscopic debridement is preferred (20). For our patient, due to great visceral pleural thickness and a large empyema cavity size, the surgeon preferred thoracotomy with open decortication.

TE is associated with high mortality and morbidity (7, 21). Larger effusions (greater than or equal to $1 / 2$ hemithorax) are more resistant to effective drainage (15). Due to large pus effusion with thickened parietal pleura, the risk of poor clinical outcome in our patient was high. The cure was considered as complete resolution of signs and symptoms, normalization of laboratory markers of infection and inflammation, and complete lung expansion with residual pleural thickening of $<2 \mathrm{~cm}$ in the CXR (5). According to this view, only partial cure was achieved in our case. Our patient was followed up for a period of seven months. In a study conducted in India, the mean duration of illness was significantly higher in the tuberculous group (mean: 171 days) compared to the nontuberculous group (mean: 20 days) (5). In our patient, TE required prolonged ICTD and surgery. The long duration of the illness and the presence of BPF are the factors associated with the adverse outcome in TE patients who have an advanced disease (6). However, recovery occurred in the present case.

\section{Conclusion}

TB patients have a protracted duration of illness. In such situations, antimicrobial therapy is often insufficient, and drainage is often unsuccessful. In our patient, recurrent infection in the pleural space needed surgical intervention (surgical drainage and decortication).

\section{Acknowledgment}

Dr. Davood MohammadZade, MD, Assistant Professor, Department of surgery.

\section{Conflict of Interest}

Authors declared no conflict of interests.

\section{References}

1. Zhai K, Lu Y, Huan-Zhong S. Tuberculous pleural effusion. J Thorac Dis. 2016; 8 (7): E48694. [DOI:10.21037/jtd.2016.05.87]

2. Tavakoli A. Incidence and prevalence of tuberculosis in Iran and neighboring countries. Zahedan J Res Med Sci. 2017 ; 19(7):e9238. [DOI:10.5812/zjrms.9238]

3. Wen P, Wei M, Han C, He Y, Wang MS. Risk factors for tuberculous empyema in pleural tuberculosis patients. Sci Rep. 2019;9(1):1-6. [DOI:10.1038/s41598-019-56140-4]

4. Akgül AG, Örki A, Örki T, Yüksel M, Arman B. Approach to empyema necessitatis. World J Surg. 2011;35(5):981-4. [DOI:10.1007/s00268-0111035-5]

5. Kundu S, Mitra S, Mukherjee S, Das S. Adult thoracic empyema: A comparative analysis of tuberculous and nontuberculous etiology in 75 patients. Lung India. 2010;27(4):196. [DOI:10.4103/0970-2113.71939]

6. Malhotra P, Aggarwal A, Agarwal R, Ray P, Gupta D, Jindal S. Clinical characteristics and outcomes of empyema thoracis in 117 patients: A comparative analysis of tuberculous vs. Nontuberculous aetiologies. Respir Med. 2007;101(3):423-30. [DOI:10.1016/j.rmed.2006.07.016] 
7. Lin MT, Wang JY, Yu CJ, Lee LN, Yang PC, Group T. Mycobacterium tuberculosis and polymorphonuclear pleural effusion: Incidence and clinical pointers. Respir Med. 2009;103(6):820-6.

[DOI:10.1016/j.rmed.2008.12.023]

8. Gui X, Xiao H. Diagnosis of tuberculosis pleurisy with adenosine deaminase (ada): A systematic review and meta-analysis. Int J Clin Exp Med. 2014;7(10):3126.

9. Sharngan S, Nair RS, Rajan D. Diagnostic cut-off of pleural fluid adenosine deaminase (ada) value in tuberculous pleural effusion. History. 2018;19:49. [DOI:10.14260/jemds/2018/191]

10. Porcel JM, Esquerda A, Bielsa S. Diagnostic performance of adenosine deaminase activity in pleural fluid: A single-center experience with over 2100 consecutive patients. Eur J Intern Med. 2010;21(5):419-23.

[DOI:10.1016/j.ejim.2010.03.011]

11. Lewinsohn DM, Leonard MK, Lobue PA, et al. Official american thoracic society/infectious diseases society of america/centers for disease control and prevention clinical practice guidelines: Diagnosis of tuberculosis in adults and children. Clin Infect Dis. 2017;64(2):e1-e33. [DOI:10.1093/cid/ciw694]

12. Fang $\mathrm{Y}$, Xiao $\mathrm{H}$, Sha $\mathrm{W}$, $\mathrm{Hu} \mathrm{H}$, You $\mathrm{X}$. Comparison of closed-chest drainage with rib resection closed drainage for treatment of chronic tuberculous empyema. J Thorac Dis. 2018;10(1):347. [DOI:10.21037/jtd.2017.11.123]

13. Acharya PR, Shah KV. Empyema thoracis: A clinical study. Ann Thorac Med. 2007;2(1):14. [DOI:10.4103/1817-1737.30356]
14. Merid Y, Woldeamanuel Y, Abebe M, Datiko D, Hailu T, Habtamu G, et al. High utility of active tuberculosis case finding in an ethiopian prison. Int J Tuberc Lung Dis. 2018;22(5):524-9. [DOI:10.5588/ijtld.17.0635]

15. Colice GL, Curtis A, Deslauriers J, et al. Medical and surgical treatment of parapneumonic effusions: An evidence-based guideline. Chest. 2000;118(4):1158-71. [DOI:10.1378/chest.118.4.1158]

16. Bhuniya S, Arunabha DC, Choudhury S, Saha I, Roy TS, Saha M. Role of therapeutic thoracentesis in tuberculous pleural effusion. Ann Thorac Med. 2012;7(4):215. [DOI:10.4103/18171737.102176]

17. De Hoyos A, Sundaresan S. Thoracic empyema. Surg Clin. 2002;82(3):643-71. [DOI:10.1016/S0039-6109(02)00022-1]

18. Sihoe AD, Shiraishi Y, Yew WW. The current role of thoracic surgery in tuberculosis management. Respirol. 2009;14(7):954-68. [DOI:10.1111/j.1440-1843.2009.01609.x]

19. Sarkar A, Sharma H, Razdan S, Kuhar S, Bansal N, Kaur G. Effect of segmental breathing exercises on chest expansion in empyema patients. Physiother Occup Ther. 2010;4(3):17.

20. Webb A, Gattinoni L. The respiratory system: Pleural cavity problems (4): Pleural effusion and haemothorax (125). Oxford textbook of critical care e-book: Oxford University Press.; 2016. p. 580 .

21. Al-Kattan KM. Management of tuberculous empyema. Eur J Cardiothorac Surg. 2000;17(3):251-4. [DOI:10.1016/S10107940(99)00370-X]

\section{How to Cite This Article:}

Allami A, Farzam S S, HosseinZadeh S. Pleural Space Complications from Tuberculous Empyema: A Case Report and Short Literature Review. J Adv Med Biomed Res. 2020; 28 (129) :218-224

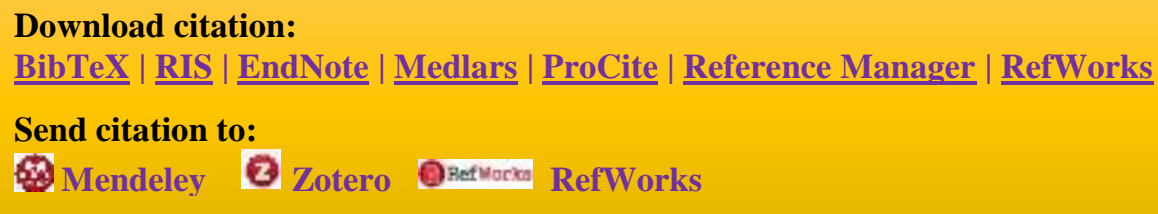

